\title{
Evaluation of a World Collection of Chickpea Germ Plasm Accessions for Resistance to Ascochyta Blight
}

\author{
M. V. REDDY, Plant Pathologist, and K. B. SINGH, Plant Breeder, International Center for Agricultural Research in \\ the Dry Areas (ICARDA), P.O. Box 5466, Aleppo, Syria
}

\begin{abstract}
Reddy, M. V., and Singh, K. B. 1984. Evaluation of a world collection of chickpea germ plasm accessions for resistance to Ascochyta blight. Plant Disease 68: 900-901.
\end{abstract}

A world collection of 9,574 desi and 3,836 kabuli germ plasm accessions of chickpea (Cicer arietinum) was evaluated for Ascochyta blight (Ascochyta rabiei) resistance at the International Center for Agricultural Research in the Dry Areas (ICARDA) research farm at Tel-Hadya near Aleppo, Syria, during four crop seasons (1978-1979 to 1981-1982). Desi accessions were obtained from the International Crops Research Institute for the Semi-Arid Tropics (ICRISAT), based in India, and the kabuli types were from ICARDA. The evaluation was carried out with artificial inoculations in the field. Promising lines from initial screening were reevaluated at Tel-Hadya and at two other sites: Lattakia, a coastal site in Syria, and Terbol, in the Beqa'a Valley of Lebanon. Lines with low disease severity during both the vegetative and podding stages were considered resistant. Eleven kabuli and six desi accessions were resistant to moderately resistant to Ascochyta blight. Another seven kabuli and 40 desi accessions were tolerant. Many lines resistant during the vegetative stage became susceptible during podding. In general, kabuli sources had better hostplant resistance than desi types. Frequency of resistance was also higher in kabuli types $(0.29 \%)$ than in desi types (0.06\%). The resistant kabuli accessions were ILC 72, ILC 196, ILC 201, ILC 202, ILC 2506, ILC 2956, ILC 3274, ILC 3279, ILC 3346, ILC 3956, and ILC 4421. The resistant desi accessions were ICC 3634, ICC 4200, ICC 4248, ICC 4368, ICC 5124, and ICC 6981.

Chickpea (Cicer arietinum L.) is an important grain legume in West Asia, the Indian subcontinent, North Africa, Ethiopia, southern Europe, and Mexico; 6.8 million tons are produced on about 10.4 million hectares (1). The average yield is estimated at $700 \mathrm{~kg} / \mathrm{ha}$. Ascochyta blight (Ascochyta rabiei (Pass.) Lab.) is one of the major causes of low and variable yields. During the past three seasons (1979-1980 to 1981-1982), Ascochyta blight epidemics occurred in Pakistan and caused nearly a $50 \%$ reduction in chickpea production (7). The disease caused serious losses in the northern states of India during the 1981 and 1982 seasons. In northern Syria, it reduced yield by nearly $30 \%$ in 1982 (2).

Development of resistant cultivars has been one of our major approaches for management of Ascochyta blight. Therefore, systematic evaluation of both kabuli (large, ramhead-shaped, beige seeds) germ plasm accessions maintained at the International Center for Agri-

Joint contribution from food legume improvement program of the International Center for Agricultural Research in the Dry Areas (ICARDA), P.O. Box 5466, Aleppo, Syria, and the International Crops Research Institute for the Semi-Arid Tropics (ICRISAT), Patancheru, P.O. Andhra Pradesh 502 324 , India.

Accepted for publication 23 April 1984.

The publication costs of this article were defrayed in part by page charge payment. This article must therefore be hereby marked "advertisement" in accordance with 18 U.S.C. $\$ 1734$ solely to indicate this fact.

() 1984 The American Phytopathological Society cultural Research in the Dry Areas (ICARDA) in Syria and desi (small, angular, black, brown, yellow, or green seeds) germ plasm accessions maintained at International Crops Research Institute for the Semi-Arid Tropics (ICRISAT) in India was initiated during the 1978-1979 season at ICARDA to identify sources of resistance to $A$. rabiei. Results from the screening of 13,410 germ plasm accessions during the past four seasons (1978-1979 to 1981-1982) are reported.

\section{MATERIALS AND METHODS}

Screening method. Germ plasm accessions were evaluated at ICARDA's research farm at Tel-Hadya, near Aleppo, Syria, under severe disease conditions during four seasons (1978-1979 to 1981-1982). In all four cropping seasons, the nursery was established in winter ( 15 November-15 December) and plants were harvested in summer (June-July). Desi germ plasm accessions $(9,574)$ were obtained from ICRISAT and kabuli germ plasm accessions $(3,836)$ were obtained from ICARDA. In the initial evaluation, 50 seeds per accession were sown in a single row $5 \mathrm{~m}$ long. The interrow and intrarow spacings were 45 and $10 \mathrm{~cm}$, respectively. A susceptible cultivar (Syrian local land race) was sown between every two to 10 test rows to serve as an indicator-spreader. A strip (1-3 m wide) of the susceptible cultivar was sown around the screening nursery to provide an additional source of inoculum.

Disease was initiated in the nursery as described earlier (6,9-11). Nurseries were inoculated during February by uniformly scattering blight-affected chickpea debris preserved from the previous season's screening nursery. During some seasons, the debris was supplemented by inoculation with spores $\left(5-15 \times 10^{4}\right.$ spores per milliliter) prepared either from freshly infected plants or fungus culture multiplied on $4 \%$ chickpea-dextrose broth. Inoculation was done in the afternoon by spraying the spore suspension with a knapsack sprayer when the plants were 3-4 mo old. During dry weather (mainly in March and April), when the crop was in flowering and podding stages, sprinkler-irrigation (slowly rotating, 60-cm-long, 9-m-radius, $3.06-\mathrm{mm}$ nozzle, $3 \mathrm{gal} / \mathrm{min}$ at $50 \mathrm{psi}$ ) for 2 hr daily for 10-15 days provided higher relative humidity.

Blight scoring scale. Disease was estimated twice: first, when the susceptible check was completely killed (late March to early April), and again, at podding (June), when disease on both vegetative parts and pods was apparent. A ninepoint scale for scoring blight severity on vegetative parts was described earlier $(9,11)$. Infections on both vegetative parts and pods were classified as follows: $1=$ immune, no infection; $2=$ highly resistant, $1-5 \% ; 3=$ resistant, $6-10 \% ; 4=$ moderately resistant, $11-15 \% ; 5=$ tolerant, $16-40 \% ; 6=$ moderately susceptible, $41-50 \% ; 7=$ moderately susceptible to susceptible, $51-75 \% ; 8=$ susceptible, $76-100 \%$ breakage of branches and pod infection; and $9=$ highly susceptible, plants killed.

Selection procedure. Lines with ratings of 5 or less were selected for further evaluation in subsequent seasons at two locations (Lattakia, Syria, and Terbol, Lebanon) besides Tel-Hayda. Results from at least $2 \mathrm{yr}$ of testing at the three locations were considered to classify the reactions of lines.

\section{RESULTS}

Blight development in all four crop seasons and at all three locations was very severe, with indicator rows usually killed by the first week of April. The weather during the 1979-1980, 1980-1981, and 1981-1982 seasons was also highly favorable for blight development. Extended rainfall and cool weather late in the season helped in the development of severe pod infection.

Kabuli and desi accessions that were resistant and moderately resistant are as 
follows. Resistant kabuli: ILC 202, ILC 4421; moderately resistant kabuli: ILC 72, ILC 196, ILC 201, ILC 2506, ILC 2956, ILC 3274, ILC 3279, ILC 3346, ILC 3856. Moderately resistant desi: ICC 3634, ICC 4200, ICC 4248, ICC 4368, ICC 5124, ICC 6981. Seven other ILC and 40 other ICC accessions were tolerant.

The prefixes ILC and ICC stand for kabuli and desi accessions, respectively. None of the 13,410 desi and kabuli germ plasm accessions evaluated was found immune or highly resistant. Kabuli types showed a higher level of resistance than desi types.

\section{DISCUSSION}

Sufficient genetic resistance to Ascochyta blight in chickpea exists that can be exploited for disease control; however, the frequency of resistant lines was very low. Only 0.29 and $0.06 \%$ of kabuli and desi accessions, respectively, were moderately resistant to resistant. Contrary to an earlier belief, both the frequency and level of resistance in kabuli types were higher than in desi types.

In both kabuli and desi types, lines such as ILC 194, ILC 2548, ICC 2160 , and ICC 5127 had a very high level of resistance during the vegetative stage but later developed serious pod infection. A few lines, such as ILC 195, were less susceptible at podding than at vegetative stages.

Earlier reports on resistant sources were based on observations made mainly during vegetative stages $(3-5,8-10)$. Use of lines possessing resistance in both the vegetative and podding stages is necessary for genetic improvement. Because high levels of pod resistance are not available in desi germ plasm, it will be necessary to incorporate kabuli sources of pod resistance.

Most resistant kabuli sources have relatively small seed (seed weight generally $25 \mathrm{~g} / 100$ ) and are latematuring. They are about $50 \%$ taller than conventional bushy types, possess a fair level of tolerance to cold, and produce reasonable yields. These resistant sources are being used both at ICARDA and ICRISAT and have also been made available to national chickpea programs in Algeria, Greece, India, Jordan, Lebanon, Morocco, Pakistan, Spain, Syria, Tunisia, and Turkey.

\section{ACKNOWLEDGMENTS}

We thank Gaby Khalaf and Samir Hadjar, Research Assistants, for their assistance.
LITERATURE CITED

1. Food and Agriculture Organization of the United Nations. 1978. Production Year Book 32:124.

2. Food Legume Improvement Program. 1982. Chickpea Pathology Progress Report. ICARDA, Aleppo, Syria.

3. Grewal, J. S., and Vir, S. 1974. Varietal resistance of gram to Ascochyta blight. Indian Phytopathol. 27:643-645.

4. Luthra, J. C., Sattar, A., and Bedi, K. S. 1938. The control of blight disease of gram by resistant types. Curr. Sci. 7:45-47.

5. Luthra, J. C., Sattar, A., and Bedi, K. S. 1943. Further studies on the control of gram blight. Indian Farming 4:413-416.

6. Nene, Y. L., Haware, M. P., and Reddy, M. V. 1981. Chickpea diseases: Resistance screening techniques. Inf. Bull. 10, ICRISAT, Patancheru A. P., India.

7. Pakistan Agricultural Research Council. 1981. Annual report (1980-81) on food legumes improvement in Pakistan. Islamabad, Pakistan.

8. Reddy, M. V., and Nene, Y. L. 1978. Screening of Cicer spp. for resistance to Ascochyta blight. (Abstr.) Proc. Int. Congr. Plant Pathol.

9. Singh, K. B., Hawtin, G. C., Nene, Y. L., and Reddy, M. V. 1981. Resistance in chickpeas to Ascochyta rabiei. Plant Dis. 65:586-587.

10. Singh, K. B., Nene, Y. L., and Reddy, M. V. 1981. International screening of chickpea for resistance to Ascochyta blight. Pages 67-87 in: Proceedings of the Workshop of Ascochyta Blight and Winter Sowing of Chickpeas. M. C. Saxena and K. B. Singh, eds. ICARDA, Aleppo, Syria.

11. Singh, K. B., and Reddy, M. V. 1983. Inheritance of resistance to Ascochyta blight in chickpea. Crop Sci. 23:9-10. 